\title{
A GESTÃO DE RISCO E A PARTICIPACCÃO DA COMUNIDADE MORADORA DE ÁREAS VULNERÁVEIS
}

\author{
Erianne Gomes dos Santos e Maria Auxiliadora Teixeira Ribeiro
}

Instituto de Psicologia Universidade Federal de Alagoas, Brasil. erianne.santos@ip.ufal.br; maria.ribeiro@ip.ufal.br

\begin{abstract}
Resumo. O risco de afundamento de um bairro, situado num município brasileiro, motivou a pesquisa de mestrado iniciada com este trabalho. Objetivamos mapear e analisar as discussões científicas produzidas, sobre a gestão de risco e a participação das pessoas que vivem em situação de vulnerabilidade socioambiental, publicadas entre 2007 e 2017. Focalizamos, nesta apresentação, a revisão da literatura acessada nas bases de dados Scientific Electronic Library Online (SciELO), Portal de Periódicos da Coordenação de Aperfeiçoamento de Pessoal de Nível Superior (CAPES) e Google Acadêmico, usando os descritores: Gestão Participativa, Participação Social, Comunidade, Áreas de risco, Socioambiental, Desastre Ambiental, Governança e Gestão Ambiental. As 41 produções científicas selecionadas e os resumos analisados possibilitaram a construção de um panorama sobre o ano, distintas áreas de conhecimento, regiões e estados do Brasil e outros países das publicações. Uma revisão dialógica levou à identificação dos temas, classificados em quatro categorias: mobilização social, perfil da população, despreparo e falta de vontade pública, fragilidade das ações de prevenção. Compreende-se o processo de implicação e articulação da sociedade e do Poder Público nesse contexto, sinalizando a possibilidade de uma gestão participativa a partir da mobilização da comunidade nas discussões que permeiam as decisões e interesses coletivos.
\end{abstract}

Palavras-chave: Gestão de Risco; Participação da Comunidade; Revisão Dialógica da Literatura.

\section{RISK MANAGEMENT AND THE PARTICIPATION OF THE COMMUNITY LIVING IN VULNERABLE AREAS}

Abstract. The risk of sinking a neighborhood, located in a Brazilian municipality, motivated the master's research initiated with this work. We aim to map and analyze the scientific discussions produced, about risk management and the participation of people living in situations of socioenvironmental vulnerability, published between 2007 and 2017. In this presentation, we focus on the review of the literature accessed in the Scientific Electronic Library databases Online (SciELO), Portal of Journals of the Coordination for the Improvement of Higher Education Personnel (CAPES) and Google Scholar, using the descriptors: Participatory Management, Social Participation, Community, Risk Areas, Socio-Environmental, Environmental Disaster, Governance and Environmental Management. The 41 selected scientific productions and the analyzed abstracts enabled the construction of an overview of the year, different areas of knowledge, regions and states of Brazil and other countries of publications. A dialogical review led to the identification of themes, classified into four categories: social mobilization, population profile, unpreparedness and lack of public will, weakness of prevention actions. The process of involving and articulating society and public authorities in this context is understood, signaling the possibility of participatory management based on the mobilization of the community in the discussions that permeate collective decisions and interests.

Keywords: Risk Management; Community Participation; Dialogic Literature Review.

\section{INTRODUÇÃO}

O risco de afundamento de um bairro, situado no município de Maceió, em Alagoas, no Nordeste do Brasil, foi fator disparador da pesquisa de mestrado, que se iniciou com este trabalho. A região é considerada área de risco, devido ao surgimento de trincas e rachaduras que "apresentam persistência de mais de um metro de comprimento e abertura 
milimétrica" e resultou na interdição de diversos imóveis e vias públicas. (Serviço Geológico do Brasil [CPRM], 2019, p. 15).

Os efeitos advindos desse sinistro afetam mais de 40 mil pessoas e são provenientes da extração mineral conduzida por uma indústria petroquímica instalada na cidade, na década de 70 (Serviço Geológico do Brasil [CPRM], 2019).

Emerge desse contexto a indagação sobre qual é, efetivamente, a participação das pessoas que vivem nas áreas vulneráveis nas ações de gerenciamento, pois apesar da formulação da Política Nacional de Proteção e Defesa Civil (PNPDEC) que institui diretrizes sobre a participação da comunidade (Lei n. 12.608, 2012), ainda existem impasses que impossibilitam gerar cooperação e articulação entre as instituições governamentais e a população (Nogueira et al., 2014).

Considerando essas informações buscou-se, em um primeiro momento, conhecer as produções científicas realizadas sobre a gestão de risco e a participação das pessoas que vivem em áreas de vulnerabilidade socioambiental, no período de 2007 a 2017, a fim de identificar quais os temas discutidos, as regiões, os países e em quais áreas do conhecimento esses estudos têm sido produzidos. Em um segundo momento, analisamos e organizamos em eixos temáticos as discussões presentes nas pesquisas acessadas em base de dados, norteadas por uma Revisão Dialógica da Literatura, que se fundamenta na perspectiva construcionista (Montuori, 2005; Walker, 2015).

\section{METODOLOGIA}

Para alcançar o objetivo deste estudo, que é o de compreender as ações de gestão de risco de áreas vulneráveis com foco na participação da comunidade, realizamos uma pesquisa qualitativa, utilizando o método da Revisão de Literatura Sistêmica e Dialógica, que se fundamenta na perspectiva construcionista (Montuori, 2005; Walker, 2015). Inicialmente, definimos a questão norteadora da pesquisa: o que há na literatura sobre a participação da comunidade na gestão de risco de áreas vulneráveis no contexto urbano, nos últimos 10 anos? Para tal, utilizamos três bases de dados: Scientific Eletronic Library Online (SciELO); Portal de Periódicos da Coordenação de Aperfeiçoamento de Pessoal de Nível Superior (CAPES) e Google Acadêmico. A busca ocorreu em agosto de 2018, em um computador de uso pessoal. Vale salientar, ainda, que essa pesquisa foi conduzida por duas pesquisadoras de maneira independente. Realizamos seis varreduras usando os seguintes cruzamentos: 
"participação social" AND "áreas de risco"; "áreas de risco" AND "comunidade"; "áreas de risco" AND "socioambiental"; "participação social" AND "desastre ambiental"; "governança" AND "gestão ambiental" AND "desastre ambiental"; "gestão participativa" AND "desastre ambiental". Utilizamos 3 parâmetros que delimitaram e orientaram a seleção dos artigos, teses e dissertações: I Parâmetro temático: produções que abordassem assuntos referentes à participação da comunidade que vive em situação de risco socioambiental no gerenciamento das ações; II Parâmetro linguístico: publicações na língua portuguesa; III Parâmetro cronológico: publicações entre 2007 e 2017. Usamos como critérios de exclusão: produções científicas repetidas, estudo de nível de graduação e trabalhos online indisponíveis.

O quadro abaixo apresenta todo o levantamento nas bases de dados, que resultou em 41 produções, sendo 29 artigos, 10 dissertações e 2 teses a serem analisadas:

Tabela 1. Síntese das publicações obtidas, excluídas e selecionadas. Maceió, 2018.

\begin{tabular}{|c|c|c|c|}
\hline Fonte & Obtidas & Excluídas & Selecionadas \\
\hline Scielo & 47 & 38 & 9 \\
\hline CAPES & 52 & 36 & 16 \\
\hline $\begin{array}{c}\text { Google } \\
\text { Acadêmico }\end{array}$ & 90 & 74 & 16 \\
\hline TOTAL GERAL & & $\mathbf{4 1}$ \\
\hline
\end{tabular}

Fonte: Autoras, 2018.

De acordo com a tabela 1 podemos identificar um grande número de exclusões, que apesar de terem sido acessadas pelas bases de dados, tais produções não tinham relação com o tema e com os objetivos deste trabalho, pois eram produções repetidas, estudos de nível de graduação ou estavam indisponíveis.

Abaixo, apresentamos um fluxograma com o detalhamento do percurso metodológico adotado nessa pesquisa.

1 - Etapa - Elaboração da pergunta norteadora

O que há na literatura sobre a participação da comunidade na gestão de risco de áreas vulneráveis no contexto urbano nos últimos 10 anos?

$2^{\text {a }}$ Etapa - Levantamento em base de dados

\begin{tabular}{l|l|l|l} 
Scielo $=9$ & CAPES $=16$ & Google Acadêmico $=16$ & TOTAL $=41$
\end{tabular}

3를 Etapa - Seleção dos dados

Parâmetros de seleção: I Parâmetro temático: produções que abordassem assuntos referentes à participação da comunidade que vive em situação de risco socioambiental no gerenciamento das 
ações; II Parâmetro linguístico: publicações na língua portuguesa; III Parâmetro cronológico: publicações entre 2007 e 2017.

Critérios de exclusão: I Produções científicas repetidas; || Estudo de nível de graduação e trabalhos online indisponíveis.

4를 Etapa - Organização e agrupamento das produções científicas

Após a leitura integral das produções científicas, as agrupamos a partir dos critérios: ano de publicação, área do conhecimento, estado/região em que foram produzidas.

5aㅡapa - Análise das produções científicas

Revisão de Literatura Sistêmica e Dialógica (Montuori, 2005; Walker, 2015).

Análise dos repertórios linguísticos (Spink, 2010; Medrado, 2013).

6 Etapa - Discussão dos resultados

Reagrupamento em 4 eixos temáticos

$7^{a}$ Etapa - Apresentação dos eixos temáticos

Fonte: Autoras, 2018.

Figura 1. Descrição das sete etapas para a elaboração da Revisão Sistêmica e Dialógica da Literatura. Maceió, 2018.

\subsection{Organização e Análise das informações}

A análise do material selecionado foi organizada em duas etapas. Na primeira, abordamos a distribuição a partir de alguns critérios: ano de publicação, área de conhecimento, estado/região e países em que foram produzidos. Na segunda, apresentamos os eixos temáticos estabelecidos conforme a análise dos repertórios presentes nas produções científicas. Adotamos a Revisão de Literatura Dialógica, que se ampara na perspectiva de Sharon Walker (2015) sob influência de Alfonso Montuori (2005), a fim de analisar as informações sobre a gestão de áreas de riscos socioambientais que encontramos nos estudos selecionados e que deram ênfase na mobilização social e na implementação das ações a serem executadas.

A característica da Revisão Dialógica é trabalhar a literatura de forma dinâmica e criativa, buscando justamente dialogar com os enunciados escritos sobre o tema, sem reduzi-los à quantificação e à sumarização das referências. Nessa perspectiva, o pesquisador participa ativamente na construção do diálogo, a partir de um olhar crítico e reflexivo sobre as produções científicas obtidas durante a revisão, de maneira a não ser um mero reprodutor de trabalhos já realizados. Essa construção traz implicações da interpretação tanto do próprio pesquisador quanto dos autores revisados (Montuori, 2005; Walker, 2015). Tal aspecto é um contraponto aos pressupostos da revisão reprodutiva, essencialmente positivista, centralizada em "quem disse o quê?". "Esta abordagem reprodutiva nos leva a 
revisões que, geralmente, são tão entediantes para ler quanto foram para escrever" (Montuori, 2005, p. 45).

Essas considerações apoiam-se na perspectiva construcionista, sobretudo na abordagem dialógica das Práticas Discursivas que têm como elemento central de análise os repertórios linguísticos, definidos enquanto unidades de construção do discurso (Spink, 2010; Medrado, 2013).

A afirmação de que esta pesquisa se apoia na perspectiva da revisão dialógica significa que buscamos compreender alguns aspectos da construção do conhecimento, norteando-nos pelo enfoque relacional da revisão e por meio das conexões, interações e distanciamentos entre esses textos, proporcionando espaços conversacionais e os organizando a partir do diálogo que proporcionam, considerando a importância de ouvir e de nos conectarmos a eles (Walker, 2015).

Essa abordagem teórico-metodológica, possibilita que se crie uma espécie de debate, situando as reflexões críticas de diversos estudos, propiciando a polifonia de vozes e posicionamentos, atentando-se, sobretudo, para a multiplicidade de versões sobre o tema.

\section{RESULTADOS}

A análise dos títulos, resumos e textos completos das 41 produções científicas permitiu que os trabalhos fossem agrupados a partir de alguns critérios: ano de publicação, área de conhecimento, estado/região e países onde foram produzidos.

Apresentamos, no quadro abaixo, um panorama da quantidade de publicações selecionadas e distribuídas entre os anos de 2007 e 2017. Optamos pela delimitação temporal correspondente a uma década, a fim de dar uma maior visibilidade ao fluxo do crescimento da produção científica referente à participação dos atores sociais na gestão de risco socioambiental.

Tabela 2. Quantidade de produções científicas sobre a participação da comunidade na gestão de riscos socioambientais por ano de publicação. Maceió, 2018.

\begin{tabular}{|c|c|c|c|c|c|c|c|c|c|c|}
\hline 2007 & 2008 & 2009 & 2010 & 2011 & 2012 & 2013 & 2014 & 2015 & 2016 & 2017 \\
\hline 1 & 1 & 4 & 3 & 1 & 4 & 6 & 5 & 7 & 6 & 3 \\
\hline
\end{tabular}

Fonte: Autoras, 2018.

Na tabela 2 podemos observar que o maior número de publicações sobre a referida temática ocorreu entre os anos de 2013-2016. Esse aspecto pode estar relacionado à criação da lei no. 12.608/12, que instituiu a Política Nacional de Proteção e Defesa Civil (PNPDEC) e dispôs sobre o Sistema Nacional de Proteção e Defesa, o que foi considerado promissor por 
proporcionar maior visibilidade aos assuntos referentes aos desastres socioambientais e às ações de gestão de risco, adquirindo relevância e visibilidade tanto no meio acadêmico como nas políticas públicas (Silva, 2017).

Identificamos, também, quais as áreas de conhecimento que contribuíram, por meio de publicações, na discussão sobre a participação da comunidade na gestão de riscos socioambientais: Saúde Coletiva/Pública (7); Ciências Sociais (6); Direito (5); Ambiente e sociedade (5); Geografia (4); Arquitetura e Urbanismo (4); Ciências Naturais (3); Engenharia Civil e Ambiental (3); Psicologia (2); Serviço Social (1); Educação e Gestão Ambiental (1).

As áreas do conhecimento da Saúde Coletiva/Pública e as Ciências Sociais são as que predominam entre as pesquisas sobre a participação da comunidade na gestão de áreas de risco sociambientais.

$\mathrm{Na}$ sequência, identificamos um quantitativo referente às regiões/estados onde foram produzidas as publicações selecionadas por meio desse levantamento de dados.

Tabela 3: Quantidade de produções científicas brasileiras por região e estado onde as pesquisas sobre a participação da comunidade na gestão de áreas de riscos socioambientais foram desenvolvidas. Maceió, 2018.

\begin{tabular}{|c|c|c|c|c|c|c|c|c|c|c|c|c|c|}
\hline \multicolumn{3}{|c|}{ Sudeste } & \multicolumn{3}{|c|}{ Sul } & \multicolumn{4}{|c|}{ Norte } & \multicolumn{2}{|c|}{ Nordeste } & \multirow{2}{*}{ 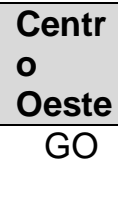 } & \multirow{2}{*}{ Total } \\
\hline $\begin{array}{l}R \\
\mathrm{~J}\end{array}$ & MG & $\begin{array}{l}\mathrm{S} \\
\mathrm{P}\end{array}$ & $\begin{array}{l}\mathrm{P} \\
\mathrm{R}\end{array}$ & $\begin{array}{l}\mathrm{S} \\
\mathrm{C}\end{array}$ & $\begin{array}{l}R \\
S\end{array}$ & AM & PA & AP & $\mathrm{RR}$ & $\mathrm{RN}$ & PB & & \\
\hline 6 & 3 & $\begin{array}{l}1 \\
5\end{array}$ & 1 & 3 & 2 & 1 & 1 & 1 & 1 & 1 & 1 & 2 & 38 \\
\hline
\end{tabular}

Fonte: Autoras, 2018.

Conforme a tabela acima, podemos observar que o Sudeste foi a região com maior número de publicações referentes à participação da comunidade na gestão de riscos socioambientais, com um total de 24 produções, com destaque para o Estado de São Paulo, onde foram realizados 15 estudos associados ao tema. De acordo com o Atlas Brasileiro de Desastres Naturais "a alta densidade demográfica aliada à ocupação desordenada em áreas de risco faz desta região uma das que mais sofrem com as adversidades atmosféricas (Centro Universitário de Estudos e Pesquisa sobre Desastres [CEPED] \& Universidade Federal de Santa Catarina [UFSC], 2013, p. 24). Na sequência, notamos que a região Sul aparece com seis, a Norte, com quatro e Nordeste e Centro Oeste com duas, totalizando 38 produções científicas brasileiras.

Identificamos, também, um quantitativo de 3 publicações de outros países: Chile (1) e Portugal (2). 
Entre as pesquisas estrangeiras, observamos que três foram realizadas na Europa e América do Sul, sendo duas em Portugal e uma no Chile, ampliando a compreensão desse tema em outros países.

Nesta segunda etapa da análise, construímos quadros no Microsoft Word, contemplando as seguintes informações: título, autores, fonte, descritores, ano de publicação, área do conhecimento, resumo e temáticas. A construção dos quadros facilitou a organização e a aproximação com o conteúdo do material de pesquisa. Essa estratégia possibilitou a definição dos eixos temáticos, uma vez que ao preencher a coluna referente às temáticas, destacamos as questões centrais de cada estudo identificadas no resumo e/ou em partes dos textos.

A partir desse processo, identificamos 11 focos de discussão, que foram organizados em eixos temáticos: Eixo 1 (mobilização social e gestão de áreas de risco socioambiental urbano), Eixo 2 (discussão teórica dos conceitos de vulnerabilidade socioambiental e resiliência), Eixo 3 (análise da implementação de projetos, conferências e políticas), Eixo 4 (descrição de espaços urbanos/não urbanos e avaliação de experiências), Eixo 5 (análise do perfil das produções científicas sobre desastres socioambientais e gestão ambiental), Eixo 6 (gestão de recursos hídricos/resíduos sólidos urbanos), Eixo 7 (sofrimento social), Eixo 8 (percepção de risco), Eixo 9 (desastres socioambientais e gênero), Eixo 10 (discussão do conceito de salubridade), Eixo 11 (educação ambiental e gestão participativa da pesca).

Os estudos correspondentes ao Eixo 1 (mobilização social e gestão de áreas de risco socioambiental urbana) são aqueles que se aproximam do interesse deste trabalho, no qual foram classificadas 12 pesquisas (8 artigos, 3 dissertações de mestrado e uma tese de doutorado) e selecionadas para serem examinadas, por meio de uma leitura minuciosa.

A partir da análise dos repertórios identificados nas pesquisas descritas no Eixo 1, agrupamos as produções científicas de acordo com os principais assuntos discutidos sobre o tema de nosso interesse, resultando em quatro subtemas: mobilização social; perfil da população; despreparo e falta de vontade pública e fragilidade das ações de prevenção.

\section{DISCUSSÃO DOS EIXOS TEMÁTICOS}

A discussão dos eixos temáticos foi realizada tal como propõe a Revisão Dialógica da Literatura, estabelecendo as conexões e interações dialógicas entre os textos, identificando 
o que eles têm em comum, articulando suas aproximações e distanciamentos (Walker, 2015).

A seguir, situaremos as discussões dos subtemas apresentados anteriormente.

\subsection{Mobilização social}

As discussões apresentadas pelas pesquisas classificadas neste subtema atentam para o fato de que as ações centralizadas no âmbito governamental, direcionadas à gestão de áreas de riscos socioambientais, têm demonstrado fragilidade em atender as necessidades das comunidades que sofrem os efeitos desses eventos. Relacionam essa dificuldade à falta de articulação com outros setores e também pela ausência da participação social na elaboração e implementação dessas ações (Jacobi, Momm-Schult, \& Bohn, 2013; Di Giulio, Serrao-Neumann, Viglio, Ferreira \& Choy, 2014; Silva, 2015).

Sob esse enfoque, os autores que se debruçam sobre a análise da intersetorialidade, no âmbito dos desastres socioambientais, propõem a ação conjunta entre Estado e sociedade civil de forma transparente e compartilhada. O envolvimento da população na gestão de áreas de riscos tende a ser mais efetiva, como possibilidade para a realização das ações de prevenção, mitigação, resposta e recuperação (Fonseca \& Burrstyn, 2009; Jacobi et al.,2013; Tavanti \& Spink, 2014; Di Giulio et al., 2014; Silva, 2015). Os diálogos proporcionados com a literatura têm apontado para a possibilidade de se caminhar na direção de uma gestão participativa, comprometida em integrar a população por meio de diversos dispositivos, como os conselhos, fóruns, comitês, reuniões em centros comunitários e ONGs, entre outros espaços que buscam fomentar a mobilização social, estreitando a relação entre governo e sociedade civil (Tavares \& Ferrante, 2009; Lipai, 2010; Sipioni \& Silva, 2013; Di Giulio et al., 2014; Tavanti \& Spink, 2014; Soledade, 2015).

Nesse contexto, a atuação de Núcleos Comunitários de Proteção e Defesa Civil (NUPDECs) é mencionada enquanto uma das ferramentas mais importantes voltadas às ações de proteção às áreas suscetíveis e/ou afetadas pelos desastres e que trabalham a partir da compreensão de que as comunidades são parceiras na redução de riscos (Tavanti \& Spink, 2014; Rosa, Mendonça, Monteiro, Souza, \& Lucena, 2015). As pesquisas defendem a mobilização da comunidade em torno dos assuntos relacionados à gestão de áreas de risco como um importante elemento para a efetividade dos resultados. O engajamento da comunidade proporciona uma nova abordagem para o enfrentamento dos riscos, a partir da 
ação conjunta e compartilhada entre sociedade civil e Estado. Tendo em vista que "a população que convive de perto estes problemas e por certo seria a mais qualificada para indicar as melhores e emergentes ações para o planejamento" (Silva, 2015, p. 86).

\subsection{Perfil da população}

Os estudos definem o perfil da população que sofre ou está suscetível aos efeitos dos riscos de desastres socioambientais, enfatizando os aspectos sociais, culturais e econômicos, permitindo uma melhor compreensão da situação vivida por essas pessoas, uma vez que as comunidades sofrem de maneiras distintas.

Nessa temática, alguns trabalhos atribuem à precariedade, às desigualdades sociais e ao desordenamento do território, status de agravantes e multiplicadores de tragédias que comprometem a capacidade de resposta social (Fonseca \& Burrstyn, 2009; Jacobi et al., 2013; Rosa et al., 2015; Silva, 2015).

Assim, a população de baixa renda está mais vulnerável aos prejuízos, devido à relação estreita entre exposição ao risco e pobreza que potencializam e ampliam seus efeitos e danos (Jacobi et al., 2013; Verde, 2013; Verde \& Schicchi, 2015; Rosa et al., 2015; Silva, 2015; Soledade, 2015). No entanto, é importante salientar que a estruturação dessa problemática não está limitada ao nível social da população, pois sua causa deve ser compreendida a partir de diversas perspectivas, abrangendo as características físicas, socioespaciais e culturais do local (Tavanti \& Spink, 2014; Rosa et al., 2015).

\subsection{Despreparo e falta de vontade pública}

Os problemas vivenciados pela população que vive em área de vulnerabilidade socioambiental são associados, nas pesquisas, à desigualdade socioeconômica, ao déficit do planejamento territorial, ao despreparo e à falta de vontade das autoridades públicas (Fonseca \& Burrstyn, 2009; Tavares \& Ferrante, 2009; Lipai, 2010; Jacobi et al., 2013; Sipioni \& Silva, 2013; Verde, 2013; Di Giulio et al., 2014; Tavanti \& Spink, 2014; Rosa et al., 2015; Silva, 2015; Soledade, 2015; Verde \& Schicchi, 2015).

Entre as três esferas de governo, o município é o principal responsável pela resolução dos problemas relacionados às questões socioambientais (Jacobi et al., 2013; Rosa et al., 2015; Verde \& Schicchi, 2015). Entretanto, de acordo com Verde (2013), na maioria das vezes, os municípios negligenciam a iminência de tragédias. 
Os governos, sobretudo os municipais, cedem aos interesses do setor imobiliário e demais elites locais, pois, em decorrência de uma "lógica clientelista", acabam abrandando as normas relacionadas ao uso do solo, isentando-os de impostos e reforçando, dessa forma, a ocupação desordenada do território e expondo a população aos riscos de desastres (Fonseca \& Burrstyn, 2009; Lipai, 2010; Jacobi et al., 2013; Soledade, 2015).

O Brasil dispõe de conhecimento técnico e profissionais capacitados para evitar ou minimizar a ocorrência de desastres, entretanto, devido à "falta de vontade pública", associada à "omissão", "insuficiência" e "impropriedade" das ações públicas, esses instrumentos não conseguem atuar de forma efetiva na gestão do espaço urbano (Jacobi et al., 2013, p. 248). Em uma situação de desastre socioambiental, a postura adotada pelas autoridades governamentais se concentra nas ações emergenciais, com o intuito de atenuar os impactos e o sofrimento da população, com receio de perder a legitimidade de seus eleitores, culminando com a falta de confiança das pessoas afetadas nos serviços governamentais responsáveis pela gestão de áreas de risco (Jacobi et al., 2013; Di Giulio et al., 2014).

Em consequência do não comprometimento das autoridades, a tendência é a recorrência dos desastres, a ampliação de sua intensidade e falta de confiança da população atingida nos serviços públicos (Jacobi et al., 2013; Di Giulio et al., 2014; Rosa et al., 2015).

\subsection{Fragilidade das ações de prevenção}

As ações de prevenção são medidas tomadas como forma de minimizar os impactos dos desastres socioambientais. São norteadas por políticas de gerenciamento e de proteção da natureza que devem ser conduzidas de forma articulada entre Poder Público e a coletividade (Soledade, 2015).

No entanto, os órgãos responsáveis pela gestão dos recursos naturais e espaço urbano se concentram no planejamento pós-desastre de caráter emergencial, devido à ausência de um planejamento voltado à prevenção desses eventos (Jacobi et al., 2013; Verde, 2013; Di Giulio et al., 2014; Tavanti \& Spink, 2014; Rosa et al., 2015).

De modo geral, o Brasil não possui tradição no enfrentamento dos desastres socioambientais, entretanto, dispõe das ações da Defesa Civil, como forma de proteger a população em casos de situações de emergência ou calamidade (Verde, 2013). 
Os diálogos apresentados no âmbito científico demonstram um cenário de fragilidade do Poder Público em lidar com a complexidade frente aos desastres socioambientais, além de um déficit das ações de prevenção dos órgãos governamentais. Uma problemática que se caracteriza pela falta de uma visão integrada e socialmente construída, marcada pela exclusão da participação social, com destaque para a culpabilização das pessoas que residem em locais frágeis e de risco, somando-se a isso a execução de ações por vezes descontextualizadas que negligenciam as características locais (Jacobi et al., 2013; Verde, 2013; Di Giulio et al., 2014; Tavanti \& Spink, 2014).

\section{CONSIDERAÇÕES FINAIS}

O método de investigação adotado nessa pesquisa privilegia técnicas qualitativas de construção de informações e é norteado pelos pressupostos teórico-metodológicos da Revisão de Literatura Dialógica, que se fundamenta na perspectiva construcionista (Montuori, 2005; Walker, 2015).

Nesse sentido, foi apresentado um panorama das discussões teóricas em torno da gestão de áreas de risco e a participação da comunidade que vive em situação de vulnerabilidade socioambiental, obtido por meio de um mapeamento de produções científicas acessadas em bases de dados, que nos permitiu conhecer as diferentes formas de contribuição científica de outras/os pesquisadoras/res sobre a referida temática, assim como, contribuiu no desenvolvimento de um posicionamento crítico e reflexivo para formular argumentos que fundamentassem a relevância do estudo (Ribeiro; Martins; Lima, 2015).

Ao avançarmos nas conexões possibilitadas por meio do nosso diálogo com a literatura, observamos que as pesquisas analisadas discutem a relevância da mobilização social para a articulação das ações, propondo, dessa forma, uma ação conjunta entre Estado e sociedade civil como uma estratégia de prevenção, mitigação, resposta e recuperação, sob a prerrogativa de que, quanto maior a implicação social, maior será a efetividade da gestão (Fonseca \& Burrstyn, 2009; Jacobi et al.,2013; Tavanti \& Spink, 2014; Di Giulio et al., 2014; Silva, 2015).

Em suma, buscamos a partir deste estudo dar visibilidade à implicação da população na gestão dos riscos socioambientais, por se tratar de uma problemática tão presente nos últimos anos e cujos efeitos têm repercutido tanto em âmbito nacional quanto internacional. Esperamos, também, a partir das discussões tecidas neste artigo, propiciar reflexões em 
termos metodológicos sobre a relevância da revisão de literatura, enquanto um elemento importante para a construção do conhecimento e o desenvolvimento do desenho de uma pesquisa científica. Enfatizamos, sobretudo, que ela seja conduzida não de uma forma meramente reprodutiva, mas a partir de um estilo de escrita relacional e prospectiva, tal como é proposto pela Revisão Dialógica de Literatura, atentando para a multiplicidade de versões sobre o tema e não para a busca de uma verdade (Montuori, 2005; Walker, 2015).

\section{REFERÊNCIAS}

Centro Universitário de Estudos e Pesquisa sobre Desastres \& Universidade Federal de Santa Catarina (2013). Atlas Brasileiro de Desastres Naturais 1991 a 2012 (2a ed., Vol. Brasil). Florianópolis.

Di Giulio, G. M., Serrao-Neumann, S., Viglio, J. E., Ferreira, L. C., \& Choy, D. L. (2014). Propostas metodológicas em pesquisas sobre risco e adaptação: experiências no Brasil e na Austrália. Ambiente \& Sociedade, 17(4), 35-54. Recuperado de: http://www.scielo.br/pdf/asoc/v17n4/a03v17n4.pdf

Fonseca, I. F., Bursztyn, M. (2009). A banalização da sustentabilidade: reflexões sobre governança ambiental em escala local. Sociedade e Estado, 24(1), 17-46. Recuperado de: http://www.scielo.br/pdf/se/v24n1/a03v24n1.pdf

Jacobi, P. R., Momm-Schlt, S.I., Bohn, N. (2013). Ação e reação: Intervenções urbanas e a atuação das instituições no pós-desastre em Blumenau (Brasil). Eure (Santiago), 243-261. Recuperado de: https://scielo.conicyt.cl/pdf/eure/v39n116/art10.pdf

Lei n. 12.608, de 10 de abril de 2012. Institui a Política Nacional de Proteção e Defesa Civil - PNPDEC. Recuperado de: http://www.planalto.gov.br/ccivil_03/_Ato2011-2014/2012/Lei/L12608.htm

Lipai, E. M. (2010). Sentidos Ambiental: O que emerge das deliberações das Conferências Nacionais de Meio Ambiente? (Dissertação de Mestrado). Universidade de Brasília - Brasília, DF, Brasil. Recuperado de: https://repositorio.unb.br/bitstream/10482/8398/1/2010_EneidaMaekawaLipai.pdf

Montuori, A. (2005). Literature review as creative inquiry: reframing scholarship as a creative process. Journal of Transformative Education, 1(10), 1-20. Recuperado de http://journals.sagepub.com/ doi/abs/10.1177/1541344605279381. doi: 10.1177/1541344605279381

Ribeiro, M. A. T.; Martins, M. H. M; Lima, J. M. (2015). A pesquisa em base de dados: como fazer? In: LANG et al. (Org.). Metodologias: pesquisas em saúde, clínica e práticas psicológicas, p. 61-83. Maceió: Edufal.

Rosa, T. S., Mendonça, M. B., Monteiro, T. G., Souza, R. M. DE, \& Lucena, R. (2015). A educação ambiental como estratégia para a redução de riscos socioambientais. Ambiente \& Sociedade, 18(3), 211 230. Recuperado de: http://www.scielo.br/pdf/asoc/v18n3/1809-4422-asoc-18-03-00211.pdf

Serviço Geológico do Brasil. (2019). Estudos sobre a instabilidade do terreno nos bairros Pinheiro, Mutange e Bebedouro, Maceió (AL) (Relatório Síntese dos Resultados nº 1). Recuperado de: http://www.cprm.gov.br/imprensa/pdf/relatoriosintese.pdf

Silva, R. D. (2015). Duas décadas de autonomia política. Estudo de caso sobre as políticas públicas de gestão ambiental urbana no município de Boa Vista/Roraima do período de 1990 a 2010 e suas repercussões para o desenvolvimento regional (Tese de Doutorado em Ciência Política). Universidade Federal do Rio Grande do Sul - Boa Vista, RR, Brasil. Recuperado de: https://www.lume.ufrgs.br/bitstream/handle/10183/116632/000965430.pdf?sequence=1 
Silva, J. C. B. (2017). Muito além da questão ambiental: discursos sobre as gestões dos riscos no contexto das emergências e desastres (Tese de Doutorado). Universidade Federal de Pernambuco - UFPE, Recife, PE, Brasil. Recuperado de:

https://repositorio.ufpe.br/bitstream/123456789/25157/1/TESE\%20Juliana\%20Catarine\%20Barbosa\%20da \%20Silva.pdf

Soledade, A. O. (2015). A educação ambiental como instrumento de participação (Dissertação de Mestrado). Universidade do Estado do Amazonas, Manaus, AM, Brasil. Recuperado de: http://tede.uea.edu.br/jspui/handle/tede/72

Sipioni, M. E., \& Silva, M. Z. e. (2013). Reflexões e interpretações sobre a participação e a representação em conselhos gestores de políticas públicas. Revista de Sociologia e Política, 21(46), 147-158. Recuperado de: http://www.scielo.br/pdf/rsocp/v21n46/09.pdf

Spink, M. J. P., Medrado, B. (2013). Produção de Sentido no Cotidiano. In Spink, M. J. P. (Org.), Práticas discursivas e produção de sentidos no cotidiano: aproximações teóricas e metodológicas. Petrópolis: Centro Edelstein de Pesquisas Sociais

Tavanti, R. M., \& Spink, M. J. (2014). Ações locais e prevenção: um estudo com adolescentes que vivem em áreas de risco socioambiental. Ambiente \& Sociedade, 17(4), 213-232. Recuperado de: http://www.scielo.br/pdf/asoc/v17n4/a13v17n4.pdf

Tavares, J., \& Ferrante, V. B. (2008). Organizações Não-Governamentais Ambientais Regionais: Entre Resistência e a Utopia, algumas Reflexões. Revista Brasileira Multidisciplinar, 11(2), 12-29. Recuperado de: http://www.revistarebram.com/index.php/revistauniara/article/view/175

Verde, P. F. (2013). Cidades históricas atingidas por tragédia ambiental: estudo de caso de São Luiz do Paraitinga (SP) (Dissertação de Mestrado). Pontifícia Universidade Católica de Campinas, Campinas, SP, Brasil. Recuperado de: http://tede.bibliotecadigital.puc-campinas.edu.br:8080/jspui/handle/tede/113

Verde, P. F., Schicchi, M. C. S. (2015). São Luiz do Paratinga: da reconstrução pós-enchente às políticas de prevenção e conservação urbana. Cadernos de Pós-Graduação em Arquitetura e Urbanismo, p. 2013.11 2013.1 25. Recuperado de:

http://editorarevistas.mackenzie.br/index.php/cpgau/article/view/Verde.2013.1/4468

Walker, S. (2015). Literature reviews: generative and transformative textual conversations. FQS, 16(3), 1-13. Recuperado de http://www.qualitative-research.net/ index.php/fqs/article/view/2291. doi: 10.17169/ fqs16.3.2291 